\title{
Outcomes from an inpatient beta-lactam allergy guideline across a large US health system
}

\author{
Kimberly G. Blumenthal MD, MSc ${ }^{1,2,3}$ (1) , Yu Li MS4, Joyce T. Hsu MD ${ }^{3,5}$, Anna R. Wolfson MD³, David N. Berkowitz \\ PharmD ${ }^{6}$, Victoria A. Carballo $\mathrm{MPH}^{7}$, Jesse M. Schwartz MD, MSc, MPH ${ }^{8}$, Kathleen A. Marquis PhD, PharmD ${ }^{5,9}$, \\ Ramy Elshaboury PharmD, BCPS-AQ ID ${ }^{10}$, Ronak G. Gandhi PharmD, BCPS ${ }^{10}$, Barbara B. Lambl MD, MPH ${ }^{11}$, \\ Monique M. Freeley RPh ${ }^{12}$, Alana Gruszecki PharmD, RPh, BCPS ${ }^{13}$, Paige G. Wickner MD, MPH ${ }^{3,5, a}$ and \\ Erica S. Shenoy MD, PhD $3,14,15$,a \\ ${ }^{1}$ Division of Rheumatology, Allergy, and Immunology, Department of Medicine, Massachusetts General Hospital, Boston, Massachusetts, ${ }^{2}$ The Mongan Institute, \\ Massachusetts General Hospital, Boston, Massachusetts, ${ }^{3} \mathrm{Harvard}$ Medical School, Boston, Massachusetts, ${ }^{4}$ University of Pittsburgh School of Medicine, \\ Pittsburgh, Pennsylvania, ${ }^{5}$ Division of Rheumatology, Allergy and Immunology, Department of Medicine, Brigham and Women's Hospital, Boston, Massachusetts, \\ ${ }^{6}$ Department of Pharmacy, Newton-Wellesley Hospital, Newton, Massachusetts, ${ }^{7}$ Partners HealthCare System, Quality, Safety, and Value, Boston, Massachusetts, \\ ${ }^{8}$ Division of Allergy and Clinical Immunology, Jewish General Hospital, McGill University, Montreal, Quebec, Canada, ${ }^{9}$ Department of Pharmacy, Brigham and \\ Women's Hospital, Boston, Massachusetts, ${ }^{10}$ Department of Pharmacy, Massachusetts General Hospital, Boston, Massachusetts, ${ }^{11}$ Division of Infectious Diseases, \\ Department of Medicine, North Shore Medical Center, Salem, Massachusetts, ${ }^{12}$ Pharmacy Department, North Shore Medical Center, Salem, Massachusetts, \\ ${ }^{13}$ Pharmacy Department, Brigham and Women's Faulkner Hospital, Boston, Massachusetts, ${ }^{14}$ Division of Infectious Diseases, Department of Medicine, \\ Massachusetts General Hospital, Boston, Massachusetts and ${ }^{15}$ Infection Control Unit, Massachusetts General Hospital, Boston, Massachusetts
}

\section{Abstract}

Objective: To assess the safety of, and subsequent allergy documentation associated with, an antimicrobial stewardship intervention consisting of test-dose challenge procedures prompted by an electronic guideline for hospitalized patients with reported $\beta$-lactam allergies.

Design: Retrospective cohort study.

Setting: Large healthcare system consisting of 2 academic and 3 community acute-care hospitals between April 2016 and December 2017.

Methods: We evaluated $\beta$-lactam antibiotic test-dose outcomes, including adverse drug reactions (ADRs), hypersensitivity reactions (HSRs), and electronic health record (EHR) allergy record updates. HSR predictors were examined using a multivariable logistic regression model. Modification of the EHR allergy record after test doses considered relevant allergy entries added, deleted, and/or specified.

Results: We identified 1,046 test-doses: 809 (77\%) to cephalosporins, 148 (14\%) to penicillins, and 89 (9\%) to carbapenems. Overall, 78 patients (7.5\%; 95\% confidence interval [CI], 5.9\%-9.2\%) had signs or symptoms of an ADR, and 40 (3.8\%; $95 \% \mathrm{CI}, 2.8 \%-5.2 \%)$ had confirmed HSRs. Most HSRs occurred at the second (ie, full-dose) step (68\%) and required no treatment beyond drug discontinuation (58\%); 3 HSR patients were treated with intramuscular epinephrine. Reported cephalosporin allergy history was associated with an increased odds of HSR (odds ratio [OR], 2.96; 95\% CI, 1.34-6.58). Allergies were updated for 474 patients (45\%), with records specified (82\%), deleted (16\%), and added (8\%).

Conclusion: This antimicrobial stewardship intervention using $\beta$-lactam test-dose procedures was safe. Overall, $3.8 \%$ of patients with $\beta$-lactam allergy histories had an HSR; cephalosporin allergy histories conferred a 3-fold increased risk. Encouraging EHR documentation might improve this safe, effective, and practical acute-care antibiotic stewardship tool.

(Received 2 January 2019; accepted 15 February 2019)

Beta-lactam antibiotic allergies, reported by up to $15 \%$ of hospitalized patients, impact acute-care antibiotic prescribing. ${ }^{1,2}$ Cephalosporins, antibiotics important to the treatment of common

Author for correspondence: Kimberly G. Blumenthal, Email: kblumenthal@mgh. harvard.edu

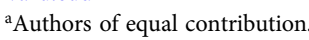

Cite this article: Blumenthal KG, et al. (2019). Outcomes from an inpatient betalactam allergy guideline across a large US health system. Infection Control \& Hospital Epidemiology, 40: 528-535, https://doi.org/10.1017/ice.2019.50 inpatient infections, are inconsistently prescribed to patients reporting penicillin allergies despite low cross reactivity. ${ }^{3-5}$ Alternatives to $\beta$-lactam antibiotics may be less effective $e^{4,6}$ and can lead to adverse sequelae for patients, most notably healthcare-associated infections such as Clostridioides difficile infection. ${ }^{7,8}$

Most patients with a documented penicillin allergy do not have clinically significant hypersensitivity and can be safely treated with penicillins and other $\beta$-lactams. ${ }^{9}$ Although penicillin allergy evaluation is encouraged by antibiotic stewardship guidelines, ${ }^{10}$

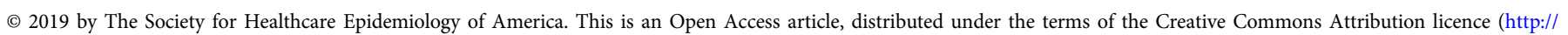
creativecommons.org/licenses/by/4.0/), which permits unrestricted re-use, distribution, and reproduction in any medium, provided the original work is properly cited. 
penicillin skin testing (PST) is operationally challenging in acutecare settings. ${ }^{11,12}$ Furthermore, many of the antibiotics generally used in hospitalized patients after a negative PST can be administered safely without preceding PST with a full-dose or test-dose challenge. ${ }^{13-15}$

The Partners HealthCare System (PHS) guideline for inpatients with $\beta$-lactam allergy histories is an antibiotic stewardship tool that includes penicillin and cephalosporin hypersensitivity pathways that direct PST when institutionally available and needed (ie, patients reporting IgE-mediated allergy symptoms to a penicillin who require a penicillin or cross-reactive cephalosporin), but it encourages direct full-dose and test-dose (ie, standardized 2-step graded) drug challenges. Prior to this study, our results demonstrated that the guideline safely increased $\beta$-lactam antibiotic use at 2 academic medical centers. ${ }^{12,14-16}$ In this study, we sought to further assess the safety of guideline-directed $\beta$-lactam antibiotic test doses after implementation of the computerized guideline throughout 5 acute-care PHS hospitals with varied resources.

\section{Methods}

\section{Computerized guideline with optional clinical decision support}

We developed $\beta$-lactam hypersensitivity pathways in 2013 at the Massachusetts General Hospital (MGH), which were modified and studied prospectively at the Brigham and Women's Hospital (BWH). ${ }^{12,14,16}$ All pathways were implemented as hospital guidelines with electronic health record (EHR) support throughout PHS acute-care sites in 2016 (Supplemental Table 1 online). ${ }^{15}$

\section{Study design overview}

We identified all PHS $\beta$-lactam antibiotic test doses performed from April 2016 through December 2017. Although $\beta$-lactam test doses were not performed at community hospital sites prior to April 2016, test doses at academic sites prior to guideline adoption occurred exclusively at the direction of an allergist. The $\beta$-lactam antibiotic test doses reviewed included those performed with and without preceding PST; PST was available at 3 sites by an allergy/immunology consultation. All patients receiving 1 or more test-dose challenge had their EHR reviewed by PHS house staff, with data entry and maintenance supported by research electronic data capture (RedCap) hosted by PHS. ${ }^{17}$

\section{Data definitions and outcomes}

The EHR-abstracted data included characteristics of the test dose (ie, drug, hospital, ordering clinician, patient care service, allergy/immunology consultation use, PST use, test-dose timing, and length of stay) and patient characteristics (ie, demographics and allergy history). Historical penicillin and cephalosporin reactions were recorded. Itching, flushing, rash, and hives were considered nonsevere cutaneous reactions; bronchospasm, shortness of breath, wheezing, anaphylaxis, angioedema, swelling, syncope, arrhythmia, hypotension, dizziness, and positive skin testing were considered severe IgE histories. Other EHR drug allergies were recorded.

The primary outcome was a hypersensitivity reaction (HSR) resulting from a $\beta$-lactam antibiotic test dose. PHS house staff reviewers recorded reaction details including timing or onset, test-dose step, symptoms and presentation, treatment, and clinical context for all possible reactions. Allergy specialist coinvestigators (K.G.B., P.G.W., J.T.H., and A.R.W.) determined whether the signs and/or symptoms were consistent with an HSR. All reactions not consistent with HSRs were considered adverse drug reactions (ADRs). For all HSRs, allergy specialists determined whether objective findings were present and whether the pathways were followed correctly. Confirmed HSRs were grouped as follows. Nonsevere cutaneous reactions included itching, flushing, rash, tingling, and urticaria; severe IgE reactions included angioedema, swelling, bronchospasm, wheezing, hypotension, and anaphylaxis; and severe delayed immunologic reactions included organ-specific reactions and severe cutaneous adverse reactions. We assessed HSRs overall by drug class, and we separately considered direct challenges (ie, challenges performed without prior PST).

Modification of the EHR allergy record after a test dose was determined by assessing whether the allergy module had a relevant allergy entry added, deleted, and/or specified (ie, additional detail was included).

\section{Statistical analysis}

We used descriptive statistics such as numbers with frequencies and medians with interquartile ranges. Univariable comparisons were made using $\chi^{2}$ and Kruskal-Wallis tests. Confidence intervals (CIs) were calculated using exact (ie, Clopper Pearson) methods. The HSR and ADR predictors were identified using multivariable logistic regression models, and we reported adjusted odds ratios (aORs) with 95\% confidence intervals (CIs). The selection of variables to include in multivariable models involved a priori knowledge of variable association with outcome. Statistical analyses were conducted using SAS version 9.4 software (SAS Institute, Cary, NC).

\section{Results}

\section{Test-dose characteristics}

From April 2016 through December 2017, 1,046 test doses to $\beta$-lactams were administered to 942 patients across 5 PHS hospitals (Table 1). Test-dose procedures were performed to penicillins $(n=148)$, cephalosporins $(n=809)$, and carbapenems $(n=89)$. Test doses were performed largely at academic sites (83\%) by house staff $(59 \%)$. The most common service performing test-dose procedures was internal medicine $(45 \%)$.

Allergy/Immunology staff were consulted for 96 (9\%) test-dose challenges administered, more often for penicillin test doses (19\%) than for carbapenem test doses (16\%) or cephalosporins (7\%; $P<.001)$. A PST prior to the test dose was performed for 38 patients $(4 \%)$, most commonly prior to penicillin test doses (13\%), compared to cephalosporins test doses (2\%) and carbapenem test doses $(0 \% ; P<.001)$.

Patients were in the hospital a median of 2 days prior to their test dose (interquartile range [IQR], 1-4 days); patients received penicillin test doses later in the hospitalization (3 days; IQR, 1-7 days) than patients who received cephalosporin (2 days; IQR, 1-4 days) or carbapenem test doses (2 days; IQR, 1-6 days; $P=.003$ ). The overall median length of stay was 10 days (IQR, 5-19 days) for patients receiving test doses.

\section{Patient characteristics}

Patients receiving test doses were mostly female (65\%) with a median age of 64 years (IQR, 51-75). Patients receiving test doses had penicillin allergy histories (96\%); $29 \%$ had cephalosporin allergy histories. Penicillin allergy histories included nonsevere 
Table 1. Test Dose and Patient Characteristics

\begin{tabular}{|c|c|}
\hline Variable & $\begin{array}{c}\text { No. }(\%) \\
(n=1,046)\end{array}$ \\
\hline \multicolumn{2}{|l|}{ Test-dose characteristics ${ }^{\mathrm{a}}$} \\
\hline \multicolumn{2}{|l|}{$\beta$-lactam drug class } \\
\hline Penicillin & $148(14)$ \\
\hline Cephalosporin & $809(77)$ \\
\hline Carbapenem & $89(9)$ \\
\hline \multicolumn{2}{|l|}{ Hospital type } \\
\hline Academic $^{b}$ & $867(83)$ \\
\hline Community $^{\mathrm{c}}$ & $179(17)$ \\
\hline \multicolumn{2}{|l|}{ Ordering provider } \\
\hline House staff & $621(59)$ \\
\hline Physician assistant & $144(14)$ \\
\hline Attending physician & $142(14)$ \\
\hline Nurse practitioner & $120(12)$ \\
\hline Unknown & $19(2)$ \\
\hline \multicolumn{2}{|l|}{ Service } \\
\hline Internal medicine ${ }^{d}$ & $469(45)$ \\
\hline Emergency department & $131(13)$ \\
\hline Surgery $^{d}$ & $126(12)$ \\
\hline Oncology & $111(11)$ \\
\hline Intensive care & $110(11)$ \\
\hline Cardiology $^{d}$ & $24(2)$ \\
\hline Neurology ${ }^{d}$ & $16(2)$ \\
\hline Obstetrics/Gynecology ${ }^{d}$ & $13(1)$ \\
\hline Pediatrics $^{\mathrm{d}}$ & $11(1)$ \\
\hline Unknown & $35(3)$ \\
\hline Allergy/Immunology consultation & $96(9)$ \\
\hline Penicillin skin test performed & $38(4)$ \\
\hline Days in hospital prior to test dose, median (IQR) & $2[1,4]$ \\
\hline Length of stay, median (IQR) & $10[5,19]$ \\
\hline Patient Characteristics $^{\mathrm{a}}$ & $n=942$ \\
\hline Female & $612(65)$ \\
\hline Age at admission, median y (IQR) & $64[51,75]$ \\
\hline Allergy to penicillin & $900(96)$ \\
\hline \multicolumn{2}{|l|}{ Penicillin reaction ${ }^{\mathrm{e}}$} \\
\hline Nonsevere cutaneous ${ }^{f}$ & $453(48)$ \\
\hline Severe IgEg & $185(20)$ \\
\hline Allergy to cephalosporin & $273(29)$ \\
\hline \multicolumn{2}{|l|}{ Cephalosporin reaction $^{h}$} \\
\hline Nonsevere cutaneous ${ }^{i}$ & $164(17)$ \\
\hline Severe $\lg \mathrm{E}^{\mathrm{j}}$ & $42(5)$ \\
\hline \multicolumn{2}{|l|}{ Other drug allergy } \\
\hline Sulfonamide antibiotics & $254(27)$ \\
\hline Opioids & $170(18)$ \\
\hline
\end{tabular}

Table 1. (Continued)

\begin{tabular}{lc}
\hline Variable & $\begin{array}{c}\text { No. }(\%) \\
(\mathrm{n}=1,046)\end{array}$ \\
\hline Fluoroquinolones & $128(14)$ \\
\hline Macrolides & $106(11)$ \\
\hline
\end{tabular}

Note. IQR, interquartile range; Ig, immunoglobulin; MGH, Massachusetts General Hospital; BWH, Brigham and Women's Hospital; NWH, Newton Wellesley Hospital; NSMC, North Shore Medical Center; BWF, Brigham and Women's Faulkner Hospital.

a Number (\%) unless otherwise specified.

${ }^{\mathrm{b}} \mathrm{MGH}$ performed 713 and BWH performed 154.

'NWH performed 89, NSMC performed 80, and BWF performed 10.

${ }^{\mathrm{d}}$ Non-intensive care.

eNumbers do not sum because patients can have $>1$ reaction. Reactions also included 214 other reactions and 197 with unknown reactions.

fNonsevere cutaneous reactions to penicillin included rash $(n=266)$, hives $(n=190)$, itching $(\mathrm{n}=35)$, and flushing $(\mathrm{n}=2)$.

gSevere IgE reactions to penicillin included anaphylaxis ( $n=78)$, angioedema $(n=48)$, swelling $(n=40)$, shortness of breath $(n=21)$, bronchospasm $(n=6)$, wheezing $(n=6)$, syncope $(n=5)$, dizziness $(n=3)$, and tested positive $(n=1)$.

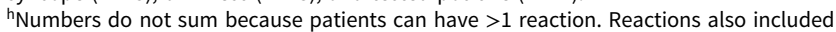
790 other reactions and 50 unknown reactions.

iNonsevere cutaneous reactions to cephalosporins included rash $(n=109)$, hives $(n=45)$, itching $(n=19)$, and flushing $(n=2)$.

jSevere IgE reactions to cephalosporins included anaphylaxis $(n=18)$, angioedema $(n=9)$, swelling $(n=8)$, shortness of breath $(n=4)$, hypotension $(n=2)$, arrhythmia $(n=2)$, and wheezing $(n=1)$.

cutaneous reactions (48\%) and severe IgE-mediated reactions (20\%). Cephalosporin allergy histories included nonsevere cutaneous reactions (17\%) and severe IgE-mediated reactions (5\%).

\section{Adverse and hypersensitivity reactions}

We identified 78 ADRs (7.5\%; 95\% CI, 5.9\%-9.2\%), of which 40 (3.8\%; 95\% CI, 2.8\%-5.2\%) were HSRs and 38 (3.6\%; $95 \%$ CI, $2.6 \%-5.0 \%$ ) were non-HSRs (eg, somatic symptoms, intolerances, or toxicities).

Most HSRs occurred after 24 hours from the initial test dose ( $\mathrm{n}=16,40 \%)$, but 14 (35\%) occurred during the 1-hour test-dose observation period (Table 2). HSRs were nonsevere cutaneous reactions $(n=25,63 \%)$. Symptoms suggestive of severe IgE-mediated reactions $(n=10,25 \%)$ and severe delayed HSRs $(n=3,8 \%)$ were also observed. Most HSRs required no treatment $(n=23$, $58 \%)$. Treatments included antihistamines $(n=16,40 \%)$, parenteral corticosteroids $(n=3,8 \%)$, and epinephrine $(n=3,8 \%)$. Objective findings had been recorded for most HSRs $(n=34$, $85 \%)$. The pathway was interpreted correctly in most cases $(n=34$, $85 \%$ ); however, the pathway was not followed correctly for 1 of the 3 patients treated with epinephrine.

An allergy to cephalosporin antibiotics (adjusted odds ratio [aOR], 2.96; 95\% CI, 1.34-6.58) was associated with increased odds of an HSR (Supplemental Table 2 online). Female sex (aOR, 1.86; 95\% CI, 1.11-3.13), allergy to cephalosporin antibiotics (aOR, 2.49; 95\% CI, 1.37- 3.13), and allergy consultation (aOR, 2.42; 95\% CI, 1.304.51) were associated with significantly increased odds of an ADR.

\section{Hypersensitivity reactions to direct test-dose challenges}

Overall, 570 penicillin allergy patients who had IgE histories or unknown histories directly challenged with cephalosporins (third, fourth, or fifth generation) or carbapenems [Fig. 1(A)]. Of the 514 patients directly challenged with third-, fourth-, or fifth-generation cephalosporins, 14 (2.7\%) had HSRs (95\% CI, 
Table 2. Hypersensitivity Reactions Resulting From $\beta$-Lactam Test-Dose Challenge Procedures

\begin{tabular}{lc}
\hline Variable & $\begin{array}{c}\text { Hypersensitivity Reactions } \\
(\mathrm{n}=40)\end{array}$ \\
\hline Reaction timing, $\mathrm{h}$ & $14(35)$ \\
\hline$\leq 1 \mathrm{~h}$ & $4(10)$ \\
\hline$>1 \mathrm{~h}$ to $<4 \mathrm{~h}$ & $2(5)$ \\
\hline$\geq 4 \mathrm{~h}$ to $<24 \mathrm{~h}$ & $16(40)$ \\
\hline$>24 \mathrm{~h}$ & $4(10)$ \\
\hline Unknown & \\
\hline Symptoms or presentation & $25(63)$ \\
\hline Nonsevere cutaneous reactions ${ }^{\mathrm{a}}$ & $10(25)$ \\
\hline Severe IgE reactions ${ }^{\mathrm{b}}$ & $3(8)$ \\
\hline Severe delayed reaction & \\
\hline Reaction treatment & \\
\hline None & $23(58)$ \\
\hline Antihistamines & $16(40)$ \\
\hline Parenteral corticosteroids & $3(8)$ \\
\hline IM epinephrine & $3(8)^{\mathrm{d}}$ \\
\hline Albuterol & $1(3)$ \\
\hline Unknown & $13(33)$ \\
\hline Objective findings & $34(85)$ \\
\hline Pathway followed and correctly & $34(85)^{\mathrm{e}}$ \\
\hline implemented & \\
\hline
\end{tabular}

Note. IM, intramuscular; PST, penicillin skin test.

ancludes rash $(n=19)$, itching $(n=6)$, hives $(n=2)$, tingling $(n=1)$. Numbers do not sum because patients can have $>1$ reaction.

Includes bronchospasm/wheezing $(n=5)$, angioedema/swelling $(n=4)$, hypotension/dizziness $(n=3)$, anaphylaxis $(n=1)$. Numbers do not sum because patients can have $>1$ reaction. 'Includes acute interstitial nephritis $(n=1)$, severe cutaneous adverse reaction $(n=1)$, and acute generalized exanthematous pustulosis $(n=1)$

${ }^{\mathrm{d} A l l} 3$ patients whose HSR required IM epinephrine treatment had cephalosporin test-dose challenges. The first patient had a history of urticaria and angioedema to penicillin and developed throat tightness, diffuse pruritus, abdominal pain, and wheezing during the cefepime full dose; IM epinephrine, hydroxyzine, and albuterol led to resolution. The second patient had a history of ampicillin anaphylaxis and received ceftriaxone by test dose and full dose uneventfully, but developed throat tightness when broadened to cefepime for

Pseudomonas spp coverage. Symptoms resolved with IM epinephrine, parenteral steroids, and antihistamines. The third patient had a history of penicillin anaphylaxis and was administered cefoxitin by test dose without prior PST. The patient experienced blurry vision, throat closing, and diffuse pruritus; symptoms resolved with IM epinephrine and diphenhydramine.

ePathway was not followed because: patient was too sick/deemed inappropriate candidate for test dose $(n=3)$, patient had active allergy symptoms $(n=2)$, or the algorithm was not correctly interpreted $(n=1)$.

$1.5 \%-4.5 \%)$. The highest HSR rate was to cefepime (4.4\%; $95 \% \mathrm{CI}$, $2.1 \%-8.0 \%)$. Of 56 patients direct challenged to carbapenems, none had HSRs.

We identified 179 patients with mild penicillin reaction histories who received direct test-dose challenges. Of 76 patients direct challenged to penicillin, 3 had HSRs (4.0\%; 95\% CI, $0.8 \%-11.1 \%)$. Of 103 patients directly challenged with cephalosporins (first or second generation), 2 patients exhibited HSRs (1.9\%; 95\% CI, 0.2\%-6.8\%) [Fig. 1(B)].

There were 135 patients with cephalosporin allergy histories directly challenged with $\beta$-lactams: 34 with penicillins (2 HSRs, 5.9\%; 95\% CI, $0.7 \%-19.7 \%$ ), 86 with cephalosporins (6 HSRs, $7.0 \%$; $95 \% \mathrm{CI}, 2.6 \%-14.6 \%$ ), and 15 with carbapenems (1 HSR, $6.7 \%$; $95 \%$ CI $0.2 \%-32.0 \%$ [Fig. $1(\mathrm{C})$ ].

\section{Updating the electronic health record allergy module}

Overall, EHR allergy sections of 474 of 1,046 patients (45\%) were updated after the $\beta$-lactam antibiotic test-dose challenge. Among the updated cases, 37 (8\%) had an allergy added, $75(16 \%)$ had an allergy deleted, and 390 (82\%) had an allergy specified (records could have $>1$ action). The PHS community hospitals updated the EHR more frequently after test doses than did PHS academic hospitals ( $54 \%$ vs $43 \%$; $P=.009$ ). Of patients who had had an allergy/immunology consultation $(n=96)$, the EHR was updated for $59(61 \%)$.

\section{Discussion}

We implemented a healthcare system-wide guideline to standardize the approach to inpatients with $\beta$-lactam allergy histories as an antibiotic stewardship tool. ${ }^{16}$ We report the outcomes of $1,046 \beta$-lactam antibiotic test-dose challenges performed by 5 diverse acute-care hospitals within a single healthcare system, largely without preceding PST (96\%). Test doses were predominantly to cephalosporin antibiotics. The ADR rate was $7.5 \%$ and the HSR rate was $3.8 \%$. Most HSRs occurred after the full-dose step and required no treatment beyond drug discontinuation. Among 10 HSRs consistent with severe IgE-mediated HSRs, 3 were treated with intramuscular epinephrine. Although a cephalosporin allergy history conferred a 3-fold increased HSR risk, HSRs to cephalosporins were infrequent in patients with specific penicillin allergy histories. Allergy records after test doses were not routinely updated.

Hospitalized patients with documented $\beta$-lactam allergies experience inferior outcomes, including treatment failures, adverse events, resistant organisms, and healthcare-associated infections. ${ }^{3,4,6-8}$ To address this problem, hospitals implemented structured allergy histories, PST, and/or comprehensive guidelines. ${ }^{11,12,18-20}$ Because PST can pose operational challenges, ${ }^{12}$ effective skin testing interventions often select inpatients for PST evaluation based on "need," such as patients on broadspectrum or nonpreferred antibiotics, ${ }^{21-23}$ or patients referred through an infectious diseases consultation. ${ }^{18,24}$ When more inclusive inpatient PST studies were attempted, only $20 \%-33 \%$ of eligible inpatients underwent testing. ${ }^{11-13}$ Our guideline uses PST only when indicated, given both the allergy history and the desired therapeutic antibiotic, and when institutionally available. This guideline applies to all adults, children, and pregnant women in all care units (eg, emergency departments, medical wards, and intensive care units). Previously, this guideline increased $\beta$-lactam use by $80 \%,{ }^{12}$ and in this study of $>1,000$ test doses, it was safe and feasible in a large, diverse healthcare system. Notably, hospitals without access to inpatient allergy/immunology consultation or PST were included, and 1 such site (Newton Wellesley Hospital, NWH), contributed almost $10 \%$ of the test doses analyzed. NWH's high test-dose volume may have been facilitated by shared MGH house staff, who had been implementing the guideline at MGH beginning in 2013, 3 years prior to other sites. Although NWH and North Shore Medical Center had consistent clinical champions since program implementation, clinical champion turnover at the Brigham and Women's Faulkner Hospital may have impacted their test-dose volume. Nevertheless, each site achieved successful implementation regardless of access to an allergist. Given that most US hospitals lack immediate allergist or PST access, our approach may be widely feasible. Indeed, some US hospitals have already adopted this approach, and pathways were incorporated into educational materials. ${ }^{19,25,26,27}$ 
A

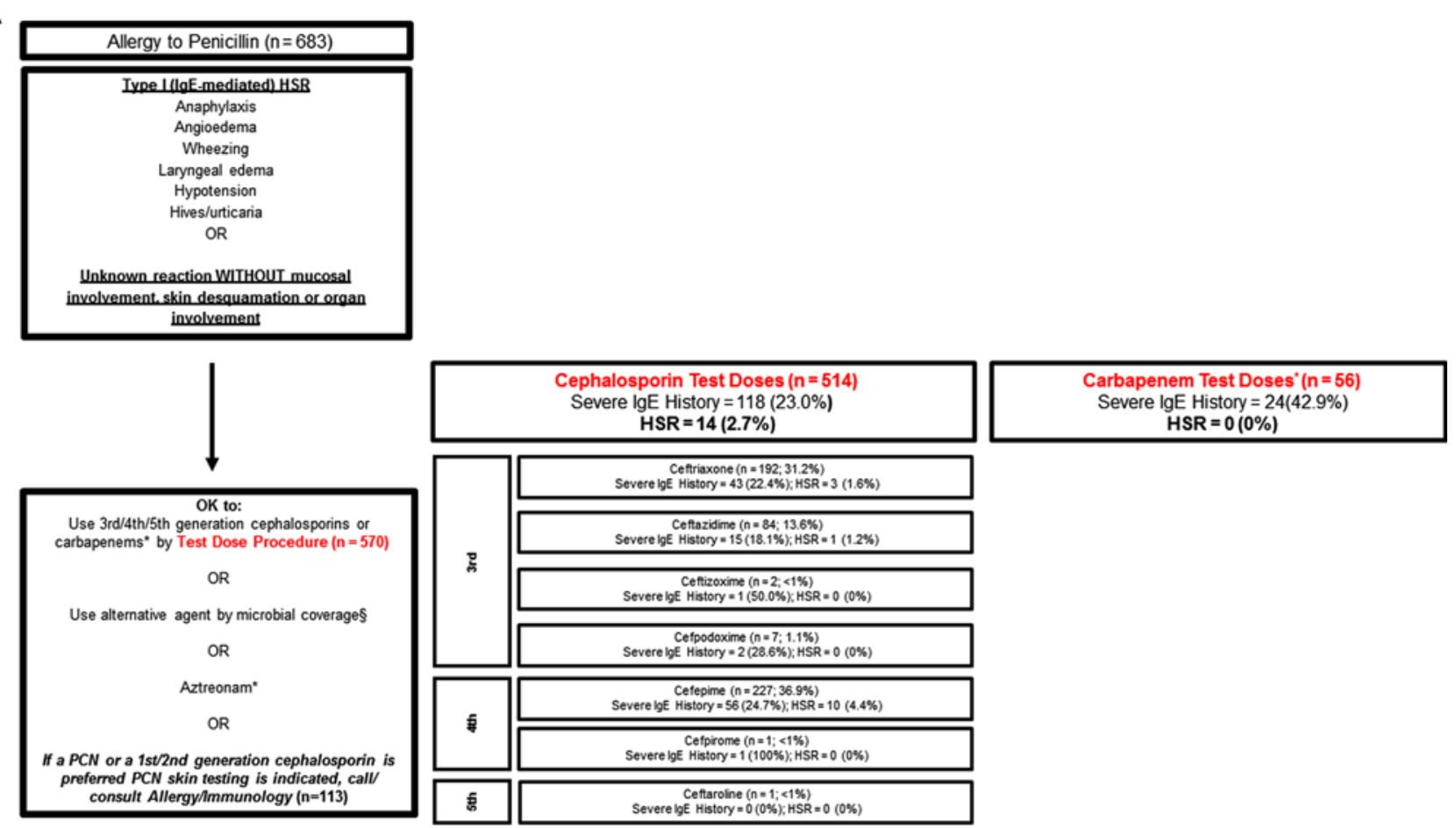

$\mathrm{B}$

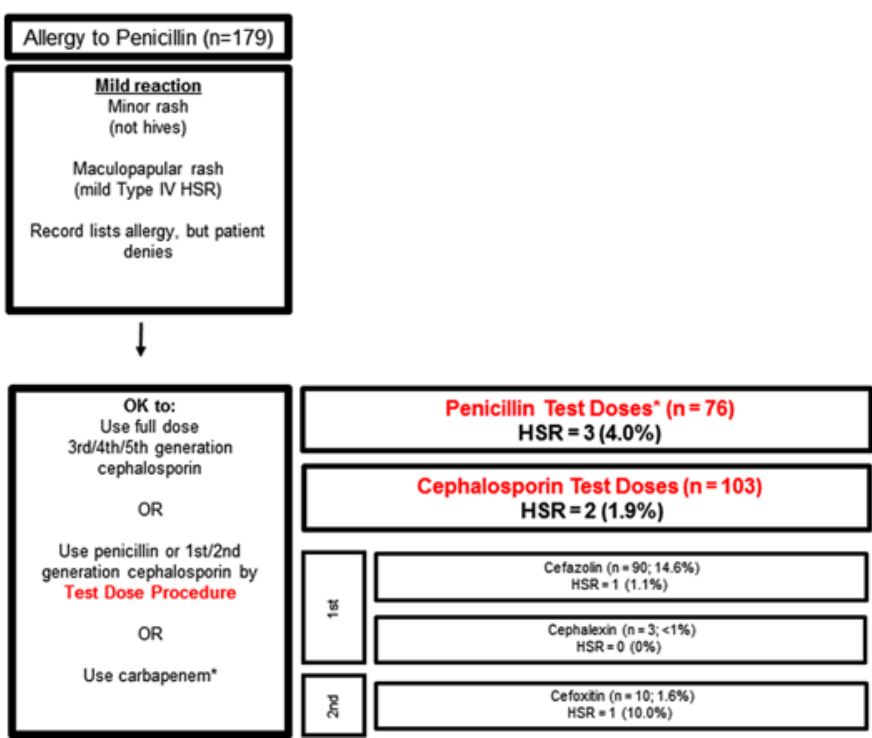

Fig. 1. Reactions from direct $\beta$-lactam antibiotic challenges. These figures provide insight into $\beta$-lactam hypersensitivity reactions (HSRs) and real-world potential cross-reactivity in acute care patients with well-characterized historical reactions. Patients exclude patients with both penicillin and cephalosporin allergy histories and those who received penicillin skin testing (PST) prior to their challenge. (A) Patients with penicillin allergy histories who received $\beta$-lactam test doses following the penicillin hypersensitivity pathway through the type 1 (IgE-mediated) HSR pathway $(n=683)$. ${ }^{*}$ Meropenem $(n=46)$, imipenem $(n=6)$, ertapenem $(n=4)$. (B) Patients with penicillin allergy histories who received $\beta$-lactam test doses following the penicillin hypersensitivity pathway through the mild HSR pathway ( $n=179)$. *Piperacillin/tazobactam $(n=26)$, ampicillin/sulbactam $(n=21)$, ampicillin $(n=13)$, amoxicillin $(n=6)$, penicillin $G(n=5)$, nafcillin $(n=4)$, amoxicillin/ clavulanic acid $(n=1)$. (C) Patients with cephalosporin allergy histories who received $\beta$-lactam test doses following the cephalosporin hypersensitivity pathway $(n=135)$. ${ }^{*}$ Piperacillin/tazobactam $(n=18)$, ampicillin/sulbactam $(n=8)$, ampicillin $(n=5)$, nafcillin $(n=2)$, penicillin $G(n=1) .{ }^{\dagger}$ Meropenem $(n=11)$, ertapenem $(n=3)$, imipenem $(n=1)$. Note. Ig, immunoglobulin; PCN, penicillin.

In this study, $7.5 \%$ of test-dose challenges resulted in an ADR. Notably, a nocebo effect (ie, noxious symptoms from a placebo drug) can occur in patients with prior drug reactions. ${ }^{28,29} \mathrm{~A}$ recent US study reported that $10 \%$ of patients who thought they were challenged to amoxicillin in an outpatient allergy practice "reacted" to the placebo. ${ }^{30}$ ADR risk factors included female sex, patients with cephalosporin allergy histories (also a significant HSR risk factor), and allergy/immunology consultation. Female sex was previously associated with higher rates of reported drug allergies/intolerances. ${ }^{31,32}$ Allergy/Immunology consultation was 


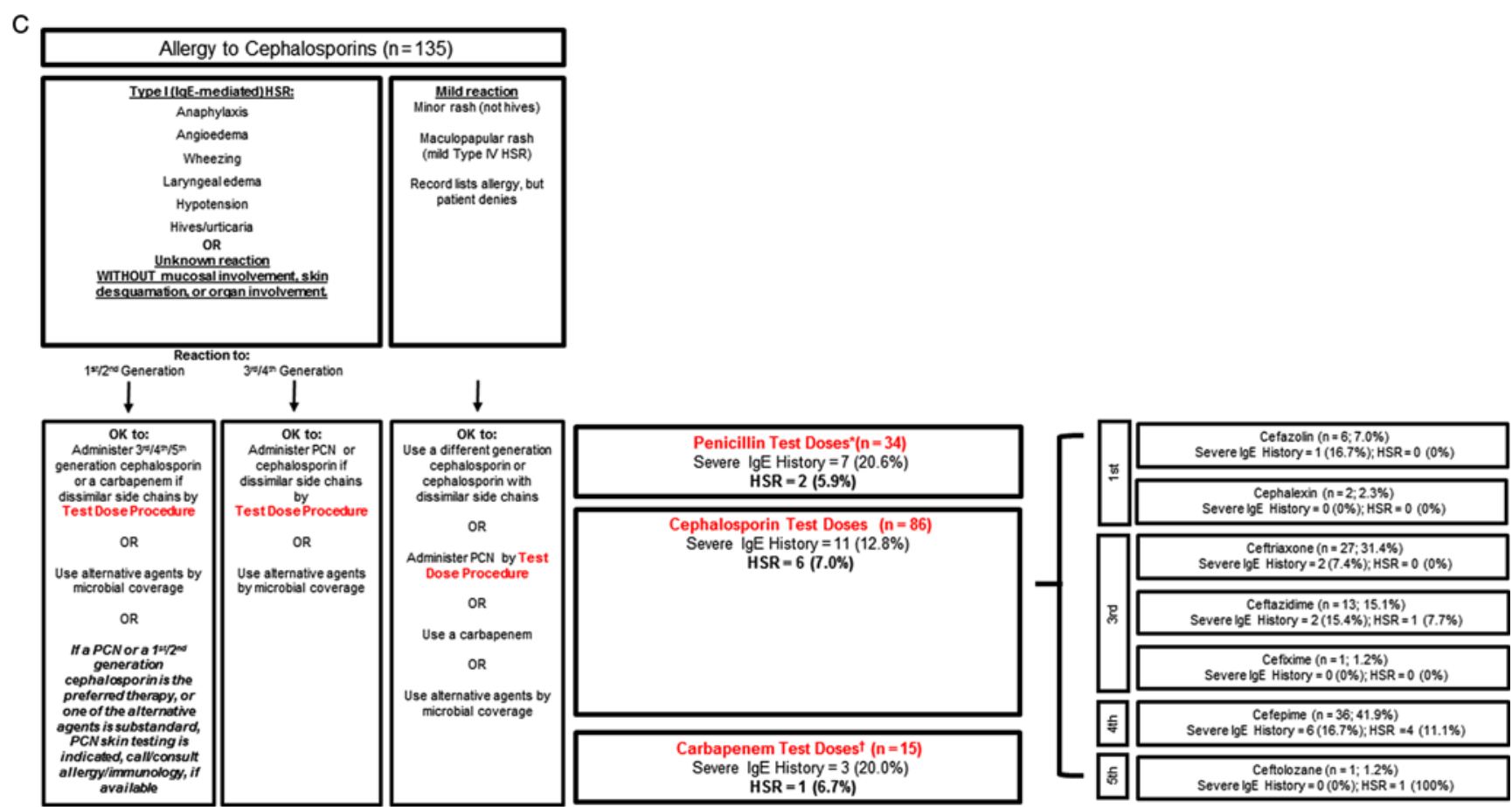

Fig. 1. Continued

associated with a higher ADR risk by design: consultations were indicated after positive challenges in locations with allergy/immunology access. The overall ADR frequency is important to consider; all patient-reported symptoms require assessment, and although reassurance may be possible for patients with only subjective symptoms, ADRs after test-dose procedures often result in drug discontinuation.

The HSR rate observed in this study was $3.8 \%$, which is similar to our prior study at $\mathrm{MGH}(3.9 \%)$ and comparable to the penicillin HSR rate observed previously by allergy specialists $(1.5 \%-2.6 \%) .^{30,33}$ Furthermore, all drug administration carries a comparable risk; hospitalized patients with infections have a baseline incidence of antibiotic allergy between $0.5 \%$ and $5.0 \%{ }^{34}$ The HSRs in this study were determined by allergist review and were largely supported by objective data. Although $\sim 25 \%$ of HSRs had signs or symptoms suggestive of a severe IgE-mediated HSR, most HSRs required no antiallergy treatment, and only 3 HSR patients were treated with epinephrine. More than one-third (35\%) of HSRs were triggered by the test dose, which may have led to those 14 patients having less severe HSRs.

This study provides insight into "real world" $\beta$-lactam cross reactivity in patients with specified IgE penicillin allergy histories, including patients with severe IgE histories. Prior observational studies of cephalosporins administered to penicillin allergy patients largely excluded patients with higher-risk penicillin allergy histories because they were selected out. ${ }^{5}$ Patients with severe IgE histories have an increased risk of true allergy and $\beta$-lactam cross reactivity. ${ }^{35,36}$ Although the later (third-, fourth-, or fifth-) generation cephalosporins overall had a low $2.6 \%$ HSR rate in patients with IgE penicillin allergy histories, the cefepime HSR rate was $4.4 \%$ with a $95 \%$ CI of up to $8.0 \%$. It remains unclear whether later-generation cephalosporins need to be initiated with a testdose challenge (many clinicians initiate these cephalosporins in penicillin allergy histories with a full dose). Of 56 carbapenem test-doses administered to patients with IgE penicillin allergy histories (including almost half with severe IgE histories), there were no HSRs, which prompts us to consider modification of the penicillin hypersensitivity pathway to indicate that carbapenems be administered by a full dose. For mild penicillin allergy histories, full-dose challenges for first- or second-generation cephalosporins are a safe modification that would facilitate the use of any cephalosporin for any patient with mild penicillin allergy histories. This change could benefit acute-care obstetric and perioperative patients where cefazolin or cefoxitin are indicated. ${ }^{3,37}$

Allergy to cephalosporin antibiotics was associated with a significant 3-fold increased odds of HSR. Documented cephalosporin allergies may more often be true hypersensitivities that occurred more recently compared to documented penicillin allergies (often "unknown" and/or remote). The cephalosporin hypersensitivity pathway may not be as accurate as the penicillin hypersensitivity pathway in this US acute-care populaton. ${ }^{38,39}$ Although a notable signal, because only 135 patients with cephalosporin allergy histories received direct cephalosporin test doses to date, more data gathering on this approach is needed prior to considering pathway modifications.

Drug allergy documentation is important for quality and safety. However, EHR allergy documentation is often incomplete and inaccurate. ${ }^{40}$ Inpatient $\beta$-lactam allergy interventions require taking an allergy history that should then be recorded in the EHR. Furthermore, results of skin tests and drug challenges should be specified in the EHR to ensure communication between providers and settings. ${ }^{41,42}$ We identified that allergy documentation was changed less than half of the time after test doses were performed. Incomplete documentation compromises the effectiveness of any allergy intervention as an antibiotic stewardship tool; $\beta$-lactams may be unnecessarily avoided or an allergy procedure might be repeated unnecessarily. Targeted alerting to the test dose prescriber may improve allergy documentation after test doses. 
Although the guideline can recommend $\beta$-lactam avoidance or administration of a full-dose $\beta$-lactam, we only reviewed $\beta$-lactams initiated by a test dose in this study. All data were abstracted and analyzed retrospectively, which can result in misclassification; however, HSR determination was rigorously determined by allergist coinvestigators. While evaluating HSRs occurring from direct challenges in patients with different allergy histories provides insight into beta-lactam cross-reactivity, patients in this study had an unknown true allergy status. Although we evaluated HSRs occurring from direct challenges in patients with different allergy histories provides insight into $\beta$-lactam cross reactivity, we were unable to obtain the true allergy statuses of patients included in this study. Finally, we report on a multisite intervention in which all hospitals are part of a single geographically localized large US healthcare system. However, non-PHS hospitals have also adopted this guideline. ${ }^{19}$

An electronic guideline with penicillin and cephalosporin hypersensitivity pathways encouraging $\beta$-lactam test-dose challenges was implemented in 5 hospitals with different resources using $1 \mathrm{EHR}$ as an antibiotic stewardship tool. The ADR and HSR rates were low, and not higher than expected given a morbid inpatient population with prior reported drug allergies. Certain test-dose challenges may be omitted given their observed safety, and caution is prudent with cephalosporin-allergy challenged inpatients. Additional efforts to improve allergy documentation are important to the success of $\beta$-lactam allergy interventions.

Supplementary material. To view supplementary material for this article, please visit https://doi.org/10.1017/ice.2019.50.

Author ORCIDs. Kimberly G. Blumenthal (D) 0000-0003-4773-9817

Acknowledgments. The authors thank Partners Penicillin Hypersensitivity Pathway team members: David W. Kubiak, PharmD, BCPS, Praveen Meka, MD, Diana Balekian, MD, MPH, and Roger P. Clark, DO. The authors thank Partners Quality, Safety, and Value and Clinical Process Improvement Leadership Program sponsors: Brian Cummings, MD and Thomas Sequist, MD, MPH. The authors thank Christian Mancini for his research assistance and Xioqing $\mathrm{Fu}, \mathrm{MS}$ for her data analysis support. Finally, the authors thank Youyang Yang, MD, Jonathan D. Paolino, MD, Arianne Baker, MD, Yasmin Islam, MD, and Robert P. McInnis, MD for serving as chart reviewers.

Financial support. This work was supported by Partners Quality, Safety, and Value and the Partners Clinical Process Improvement Leadership Program. Dr. Blumenthal receives career development support from the National Institutes of Health (grant no. K01AI125631), the American Academy of Allergy Asthma and Immunology Foundation, and the MGH Claflin Distinguished Scholar Award. The content is solely the responsibility of the authors and does not necessarily represent the official views of the National Institutes of Health.

Conflicts of interest. Dr Blumenthal reports royalties from UpToDate and honoraria from New England Society of Allergy, Dartmouth Medical School, and Vanderbilt Medical School, outside the submitted work. Drs Blumenthal and Shenoy report a licensed clinical decision support tool for $\beta$-lactam allergy evaluation that is used institutionally at Partners HealthCare System.

\section{References}

1. Conway EL, Lin K, Sellick JA, et al. Impact of penicillin allergy on time to first dose of antimicrobial therapy and clinical outcomes. Clin Ther 2017; 39:2276-2283.

2. Lee CE, Zembower TR, Fotis MA, et al. The incidence of antimicrobial allergies in hospitalized patients: implications regarding prescribing patterns an demerging bacterial resistance. Arch Intern Med 2000; 160:2819-2822.
3. Blumenthal KG, Ryan EE, Li Y, Lee H, Kuhlen JL, Shenoy ES. The impact of a reported penicillin allergy on surgical site infection risk. Clin Infect Dis 2018;66:329-336.

4. Jeffres MN, Narayanan PP, Shuster JE, Schramm GE. Consequences of avoiding beta-lactams in patients with beta-lactam allergies. J Allergy Clin Immunol Pract 2016;137:1148-1153.

5. Macy E, Blumenthal KG. Are cephalosporins safe for use in penicillin allergy without prior allergy evaluation? J Allergy Clin Immunol Pract 2018; 6:82-89.

6. Blumenthal KG, Shenoy ES, Huang M, et al. The impact of reporting a prior penicillin allergy on the treatment of methicillin-sensitive Staphylococcus aureus bacteremia. PLoS One 2016;11:e159406.

7. Macy E, Contreras R. Health care use and serious infection prevalence associated with penicillin "allergy" in hospitalized patients: a cohort study. J Allergy Clin Immunol 2014;133:790-796.

8. Blumenthal KG, Lu N, Zhang Y, Li Y, Walensky RP, Choi HK. Risk of meticillin resistant Staphylococcus aureus and Clostridium difficile in patients with a documented penicillin allergy: population based matched cohort study. BMJ 2018;361:k2400.

9. Sacco KA, Bates A, Brigham TJ, Imam JS, Burton MC. Clinical outcomes following inpatient penicillin allergy testing: a systematic review and meta-analysis. Allergy 2017;72:1288-1296.

10. Barlam TF, Cosgrove SE, Abbo LM, et al. Implementing an antibiotic stewardship program: guidelines by the Infectious Diseases Society of America and the Society for Healthcare Epidemiology of America. Clin Infect Dis 2016;62:e51-e77.

11. Chen JR, Tarver SA, Alvarez KS, Tran T, Khan DA. A proactive approach to penicillin allergy testing in hospitalized patients. J Allergy Clin Immunol Pract 2017;5:686-693.

12. Blumenthal KG, Wickner PG, Hurwitz $S$, et al. Tackling inpatient penicillin allergies: assessing tools for antimicrobial stewardship. J Allergy Clin Immunol 2017;140:154-161.

13. Rimawi RH, Cook PP, Gooch M, et al. The impact of penicillin skin testing on clinical practice and antimicrobial stewardship. J Hosp Med 2013;8:341-345.

14. Blumenthal KG, Shenoy ES, Hurwitz S, Varughese CA, Hooper DC, Banerji A. Effect of a drug allergy educational program and antibiotic prescribing guideline on inpatient clinical providers' antibiotic prescribing knowledge. J Allergy Clin Immunol Pract 2014;2:407-413.

15. Blumenthal KG, Shenoy ES, Wolfson AR, et al. Addressing inpatient betalactam allergies: a multihospital implementation. J Allergy Clin Immunol Pract 2017;5:616-625.

16. Blumenthal KG, Shenoy ES, Varughese CA, Hurwitz S, Hooper DC, Banerji A. Impact of a clinical guideline for prescribing antibiotics to inpatients reporting penicillin or cephalosporin allergy. Ann Allergy Asthma Immunol 2015;115:294-300.

17. Harris PA, Taylor R, Thielke R, Payne J, Gonzalez N, Conde JG. Research electronic data capture (REDCap)-a metadata-driven methodology and workflow process for providing translational research informatics support. J Biomed Inform 2009;42:377-381.

18. Heil EL, Bork JT, Schmalzle SA, et al. Implementation of an infectious disease fellow-managed penicillin allergy skin testing service. Open Forum Infect Dis 2016;3(3):ofw155.

19. Sacco KA, Cochran BP, Epps K, Parkulo M, Gonzalez-Estrada A. Inpatient beta-lactam test-dose protocol promotes antimicrobial stewardship in patients with history of penicillin allergy. Ann Allergy Asthma Immunol 2019;122:184-188.

20. Vaisman A, McCready J, Hicks S, Powis J. Optimizing preoperative prophylaxis in patients with reported beta-lactam allergy: A novel extension of antimicrobial stewardship. J Antimicrob Chemother 2017; 72:2657-2660.

21. Swearingen SM, White C, Weidert S, Hinds M, Narro JP, Guarascio AJ. A multidimensional antimicrobial stewardship intervention targeting aztreonam use in patients with a reported penicillin allergy. Int J Clin Pharm 2016;38:213-217.

22. Staicu ML, Brundige ML, Ramsey A, et al. Implementation of a penicillin allergy screening tool to optimize aztreonam use. Am J Health Syst Pharm 2016;73:298-306. 
23. Leis JA, Palmay L, Ho G, et al. Point-of-care beta-lactam allergy skin testing by antimicrobial stewardship programs: a pragmatic multicenter prospective evaluation. Clin Infect Dis 2017;65:1059-1065.

24. Wall GC, Peters L, Leaders CB, Wille JA. Pharmacist-managed service providing penicillin allergy skin tests. Am J Health Syst Pharm 2004;61:1271-1275.

25. Wolfe M, Schoen J, Bergman S, May S, Van Schooneveld T. Penicillin Allergy Guidance Document. https://www.nebraskamed.com/sites/ default/files/documents/for-providers/asp/penicillin-allergy-guidance.pdf. Accessed June 21, 2018.

26. Vaisman A, McCready J, Powis J. Clarifying a "penicillin" allergy: a teachable moment. JAMA Intern Med 2017;177:269-270.

27. Blumenthal KG, Solensky R. Choice of antibiotics in penicillin-allergic hospitalized patients. UpToDate website. https://www.uptodate.com/ contents/choice-of-antibiotics-in-penicillinallergic-hospitalized-patients. Accessed September 6, 2018.

28. Liccardi G, Senna G, Russo M, et al. Evaluation of the nocebo effect during oral challenge in patients with adverse drug reactions. J Investig Allergol Clin Immunol 2004;14:104-107.

29. Lombardi C, Gargioni S, Canonica GW, Passalacqua G. The nocebo effect during oral challenge in subjects with adverse drug reactions. Eur Ann Allergy Clin Immunol 2008;40:138-141.

30. Iammatteo M, Alvarez Arango S, Ferastraoaru D, et al. Safety and outcomes of oral graded challenges to amoxicillin without prior skin testing. J Allergy Clin Immunol Pract 2019;7:236-243.

31. Blumenthal KG, Li Y, Acker WW, et al. Multiple drug intolerance syndrome and multiple drug allergy syndrome: epidemiology and associations with anxiety and depression. Allergy 2018;73:2012-2023.

32. Macy E, Ho NJ. Multiple drug intolerance syndrome: prevalence, clinical characteristics, and management. Ann Allergy Asthma Immunol 2012;108:88-93.

33. Tucker MH, Lomas CM, Ramchandar N, Waldram JD. Amoxicillin challenge without penicillin skin testing in evaluation of penicillin allergy in a cohort of Marine recruits. J Allergy Clin Immunol Pract 2017;5:813-815.

34. Bigby M, Jick S, Jick H, Arndt K. Drug-induced cutaneous reactions. A report from the Boston Collaborative Drug Surveillance Program on 15,438 consecutive inpatients, 1975 to 1982. JAMA 1986;256: 3358-3363.

35. Chiriac AM, Wang Y, Schrijvers R, et al. Designing predictive models for beta-lactam allergy using the drug allergy and hypersensitivity database. J Allergy Clin Immunol Pract 2018;6:139-148.

36. Romano A, Valluzzi RL, Caruso C, Maggioletti M, Quaratino D, Gaeta F. Cross-reactivity and tolerability of cephalosporins in patients with IgE-mediated hypersensitivity to penicillins. J Allergy Clin Immunol Pract 2018;6:1662-1672.

37. Macy E. Penicillin skin testing in pregnant women with a history of penicillin allergy and group B Streptococcus colonization. Ann Allergy Asthma Immunol 2006;97:164-168.

38. Romano A, Gaeta F, Valluzzi RL, et al. IgE-mediated hypersensitivity to cephalosporins: cross-reactivity and tolerability of alternative cephalosporins. J Allergy Clin Immunol 2015;136:685-691.

39. Zagursky RJ, Pichichero ME. Cross-reactivity in beta-lactam allergy. J Allergy Clin Immunol Pract 2018;6:72-81.

40. Blumenthal KG, Park MA, Macy EM. Redesigning the allergy module of the electronic health record. Ann Allergy Asthma Immunol 2016; 117:126-131.

41. Oliver WD, Heil EL, Thom KA, Martinez JP, Hayes BD. Allergy profile should be updated after uneventful administration of a penicillin or penicillin-related antibiotic to a patient with penicillin allergy. J Allergy Clin Immunol Pract 2017;5:184-186.

42. Bouwmeester MC, Laberge N, Bussieres JF, Lebel D, Bailey B, Harel F. Program to remove incorrect allergy documentation in pediatrics medical records. Am J Health Syst Pharm 2001;58:1722-1727. 